\title{
Conservative Semi-Lagrangian solvers on mapped meshes
}

M. Mehrenberger, M. Bergot, V. Grandgirard, G. Latu, H. Sellama, E. Sonnendrücker

Université de Strasbourg, INRIA Grand Est, Projet CALVI, ANR GYPSI

ICOPS 2012 


\section{Outline}

- Introduction

- Review of the methods on cartesian grids

- BSL, FSL and CSL 


\section{GYSELA}

GYSELA code (GYrokinetic SEmi LAgrangian), CEA Cadarache

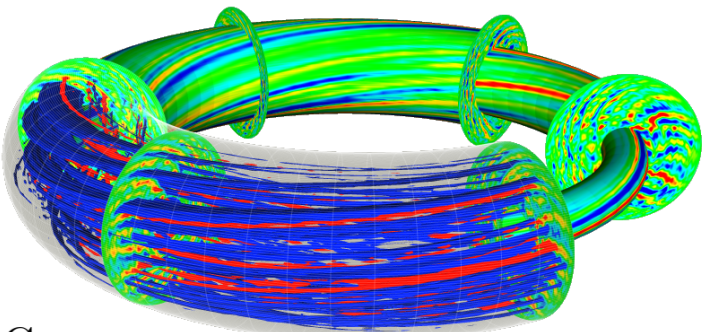

GYSELA

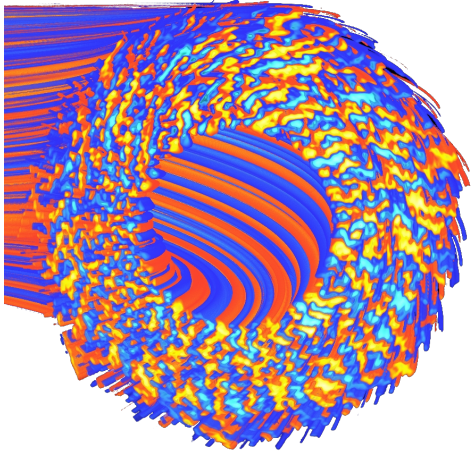

(Courtesy V. Grandgirard) $5 D$ mesh of $272 \cdot 10^{9}$ points. 31 days on 8192 processors 


\section{SeLaLib}

SEmi LAgrangian Llbrary

Goal

modular library for the gyrokinetic simulation model by a semi-Lagrangian method Support

- Large scale Initiative Fusion of INRIA

- ANR Project GYPSI (2010-2014)

- INRIA CALVI Project

- Collaboration with CEA Cadarache 


\section{Vlasov equation}

Distribution function $f(t, x, v)$ solution of the Vlasov equation $f(t, x, v) \mathrm{d} x \mathrm{~d} v$ represents the probability of finding particules in a volume element $\mathrm{d} x \mathrm{~d} v$ at time $t$ at point $(x, v)$ (position, velocity)

$$
\partial_{t} f+v \cdot \nabla_{x} f+F(t, x) \cdot \nabla_{v} f=0
$$

- Transport equation

- Non linearity through the field $F$ which depends on $f$ (Poisson, Maxwell)

- Description of the dynamic of charged particles in a plasma 


\section{Vlasov-Poisson $(1 \mathrm{D} \times 1 \mathrm{D})$}

\section{Vlasov-Poisson system}

$$
\partial_{t} f(t, x, v)+v \partial_{x} f(t, x, v)+E(t, x) \partial_{v} f(t, x, v)=0,
$$

where the field $E$ is solution of the Poisson equation

$$
\partial_{x} E(t, x)=\int_{\mathbb{R}} f(t, x, v) d v-1
$$

with zero mean condition $\left(\int_{0}^{L} E(t, x) d x=0\right)$

$\Rightarrow$ Simplified model ; first plasmas test cases

$\Rightarrow$ Smooth solution but development of small scales 


\section{Guiding center model $(1 \mathrm{D} \times 1 \mathrm{D})$}

\section{The guiding center model}

$$
\partial_{t} f(t, x, y)+\partial_{x}\left(E_{y}(t, x, y) f(t, x, v)+\partial_{y}\left(-E_{x}(t, x, y) f(t, x, y)\right)=0,\right.
$$

where the field $E=\left(E_{x}, E_{y}\right)=-\nabla \Phi$ is solution of the Poisson equation

$$
-\Delta \Phi=f(t, x, y)
$$




\section{GYSELA model 5D}

1. The variables

$$
f=f\left(t, r, \theta, \phi, v_{/ /}, \mu\right)
$$

2. Transport equation in $r, \theta, \phi, v_{/ /}$, loop over $\mu$

3. Quasi neutral equation, similar to a 3D Poisson equation

4. Gyroaverage operator : average on circle of radius depending on $\mu$ 


\section{Need of curvilinear meshes}

1. Geometry of the tokamak

2. Mesh along field lines, invariants

3. Design of robust methods

\section{Difficulties}

1. Definition of the mapping

2. Generalization of methods first defined on cartesian meshes

3. Keep if possible good properties valid on cartesian meshes 


\section{The constant advection case}

1. Typically for Strang splitting of Vlasov-Poisson

2. High order in space needed

3. Equivalence of conservative and advective form

4. No CFL restriction

5. Higher order splitting in time possible for Vlasov-Poisson

6. FFT type implementation possible

7. Little diffusion better than dispersion

8. Slope limiters possible (useful ?) 


\section{Keen testcase with SPL(7)}

Some numerical results on the Keen code (Afeyan, Crouseilles, Sonnendrücker, 2012)

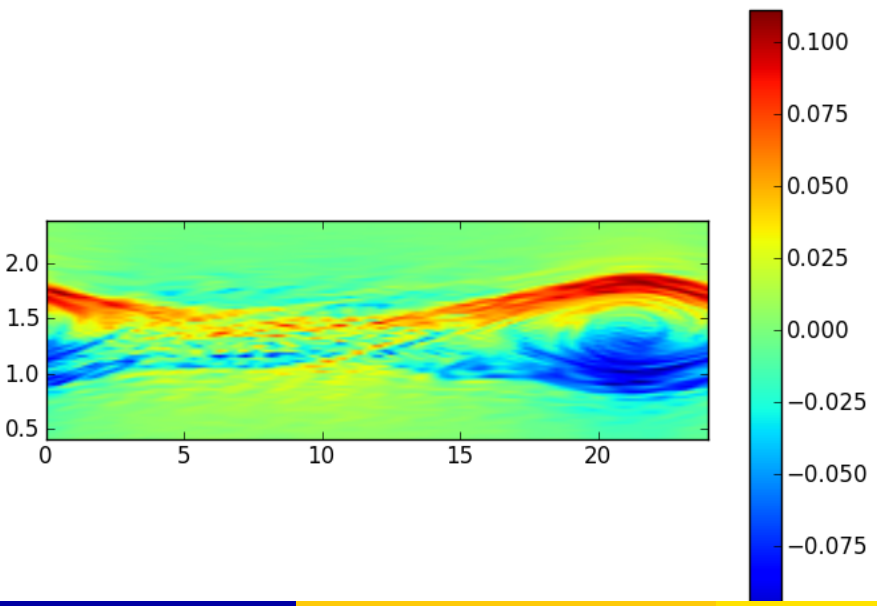




\section{Keen testcase with LAG(17)}

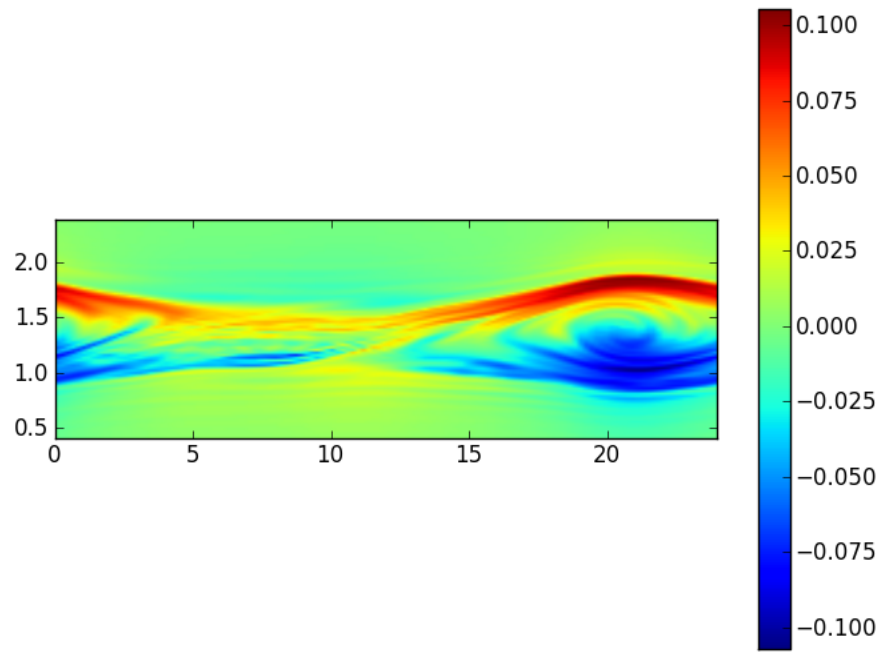




\section{Keen testcase with SPL(7)}

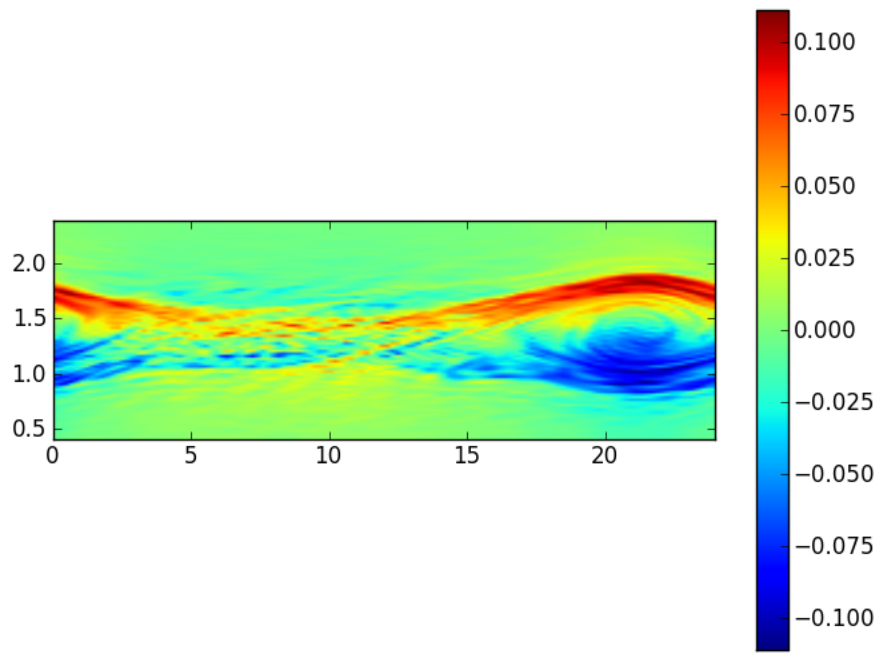




\section{Keen testcase with $\operatorname{SPL}(7) \Delta t / 10$}

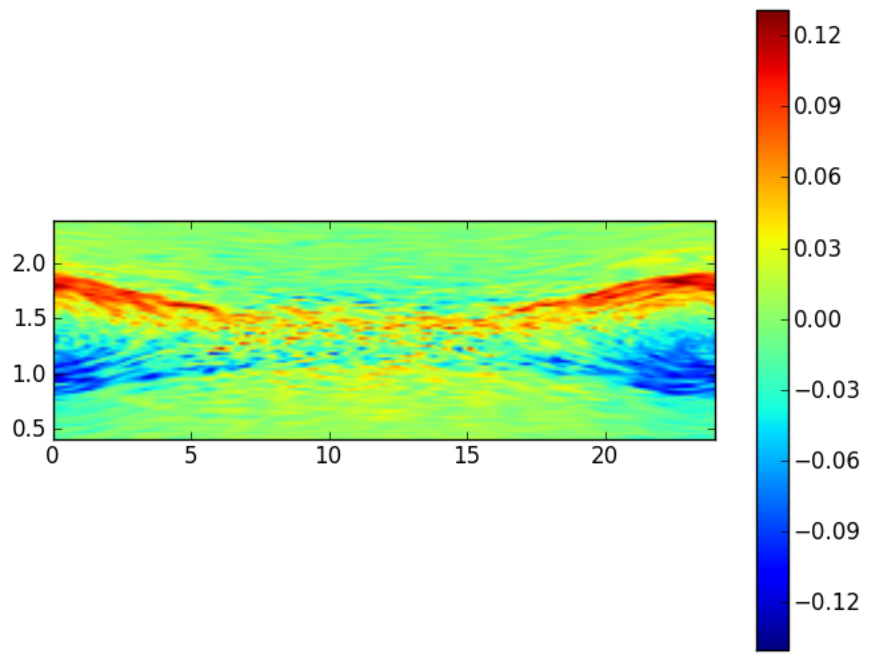




\section{Keen testcase with LAG(17) $\Delta t / 10$}

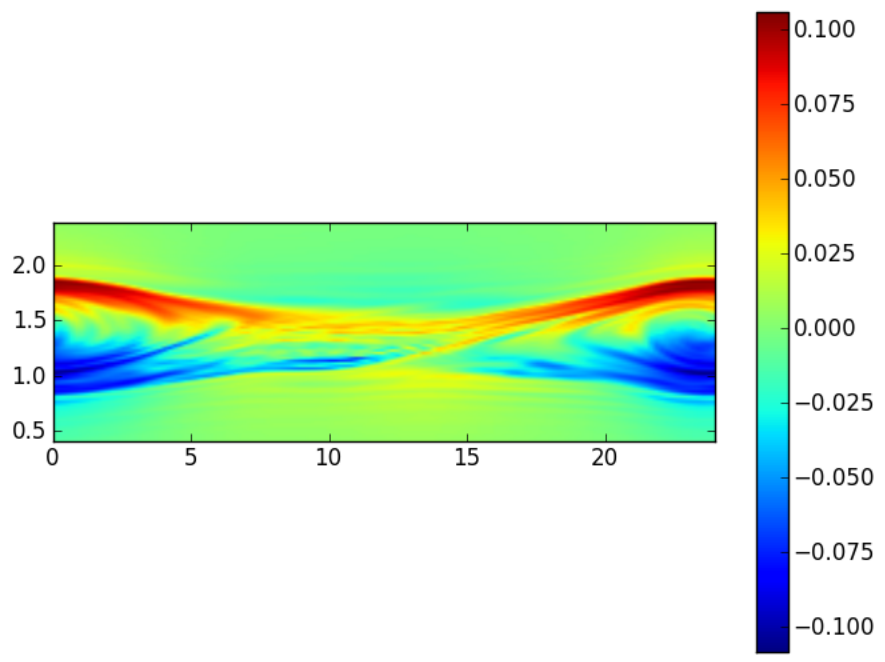




\section{Keen testcase with LAG(17)}

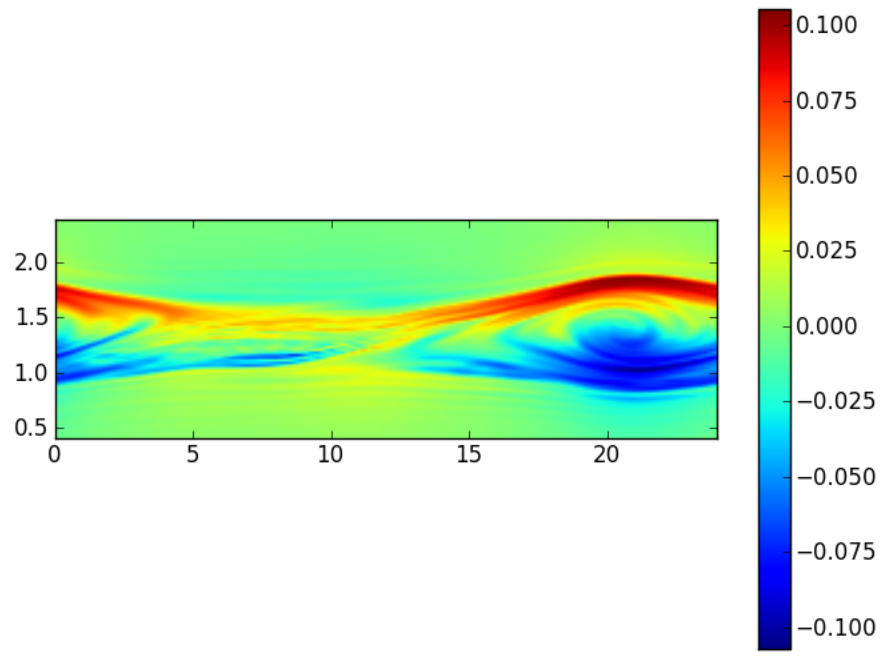




\section{The non constant advection case}

1. Typically for the guiding center model

2. History in the team

- BSL [Sonnendrücker et al., 98]

- CSL with 1d splitting on conservative form [Filbet et al., 2001, Crouseilles et al., 2010]

- FSL [Respaud et al., 2010]

5. Mass conservation vs divergence free property

6. Distinction between point values and cell averages 


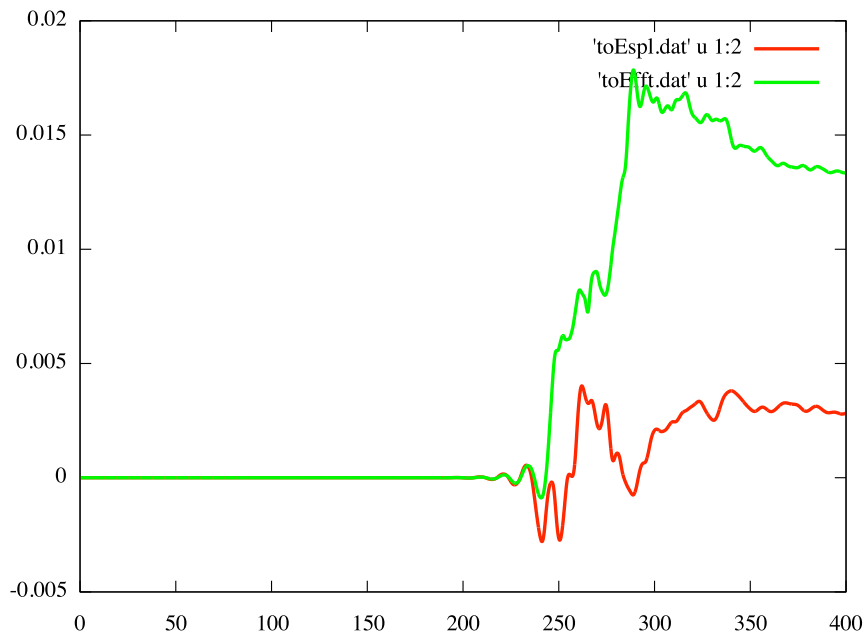

FIGURE: conservation of mass for Fourier (green) and spline (red) $d t=0.1$ (middle) and $d t=0.05$ (right) versus time. $N x=N y=64$, a guiding center testcase 


\section{Exemple in GYSELA}

PSM [Braeunig et al., 2010]
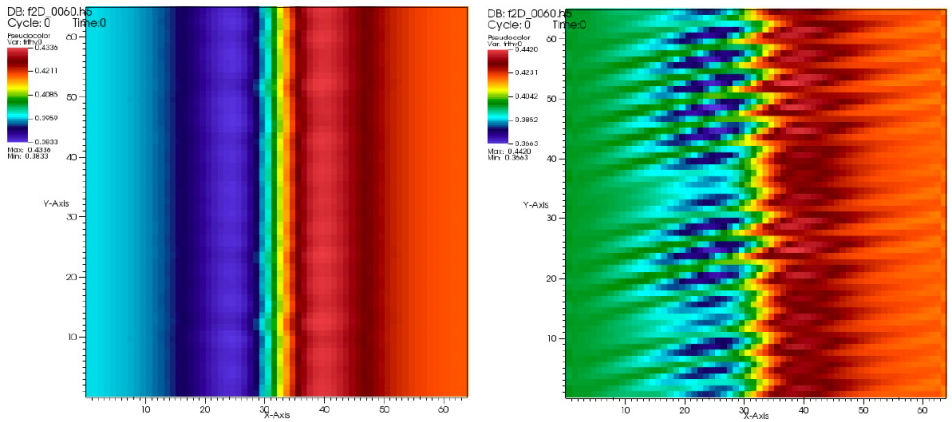

FIGURE: CSL with or without first order divergence free compatibility of the electric field 


\section{Properties to fulfill}

1. High order accuracy; problematic of cell average and point values

2. Mass conservation

3. Divergence free condition

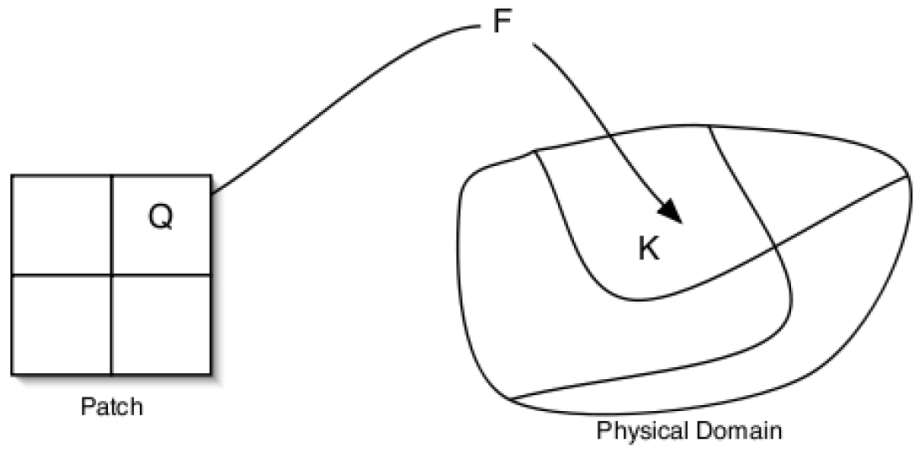

Mapping leads to non constant advection case

$\Rightarrow$ similar problems 


\section{FSL and BSL in the curvilinear case}

\section{Conservative BSL Method}

Decomposed function

$$
\tilde{f}^{n}\left(\eta^{1}, \eta^{2}\right) \approx \sum_{k, l} \tilde{\omega}_{k, l}^{n} S_{k}\left(\eta^{1}\right) S_{l}\left(\eta^{2}\right)
$$

Updating step (interpolation)

$$
\tilde{f}_{i, j}^{n+1}=\sum_{k, l} \tilde{\omega}_{k, l}^{n} S_{k}\left(\eta_{i}^{1^{*}}\right) S_{l}\left(\eta_{j}^{2^{*}}\right)
$$

\section{Non-Conservative BSL Method}

Decomposed function

$$
\bar{f}^{n}\left(\eta^{1}, \eta^{2}\right) \approx \sum_{k, l} \bar{\omega}_{k, l}^{n} S_{k}\left(\eta^{1}\right) S_{l}\left(\eta^{2}\right)
$$

Updating step (interpolation)

$$
\tilde{f}_{i, j}^{n+1}=\frac{1}{J_{i j}} \sum_{k, l} \bar{\omega}_{k, l}^{n} S_{k}\left(\eta_{i}^{1^{*}}\right) S_{l}\left(\eta_{j}^{2^{*}}\right)
$$

where $S_{q}\left(\eta_{p}\right)=S\left(\eta_{p}-\eta_{q}\right)$.

\section{Conservative FSL Method}

Decomposed function

$$
\bar{f}^{n}\left(\eta^{1}, \eta^{2}\right) \approx \sum_{k, l} \bar{\omega}_{k, l}^{n} S_{k}\left(\eta^{1}\right) S_{l}\left(\eta^{2}\right)
$$

Updating step (deposition)

$$
\tilde{f}_{i, j}^{n+1}=\frac{1}{J_{i j}} \sum_{k, l} \bar{\omega}_{k, l}^{n} S_{i}\left(\eta_{k}^{1^{*}}\right) S_{j}\left(\eta_{l}^{2^{*}}\right)
$$

\section{Non-Conservative FSL Method}

Decomposed function

$$
\tilde{f}^{n}\left(\eta^{1}, \eta^{2}\right) \approx \sum_{k, l} \tilde{\omega}_{k, l}^{n} S_{k}\left(\eta^{1}\right) S_{l}\left(\eta^{2}\right)
$$

Updating step (deposition)

$$
f_{i, j}^{n+1}=\sum_{k, l} \tilde{\omega}_{k, l}^{n} S_{i}\left(\eta_{k}^{1^{*}}\right) S_{j}\left(\eta_{l}^{2^{*}}\right)
$$




\section{Case of rotation polar coordinates $\Delta t=0.1 \Delta x$}

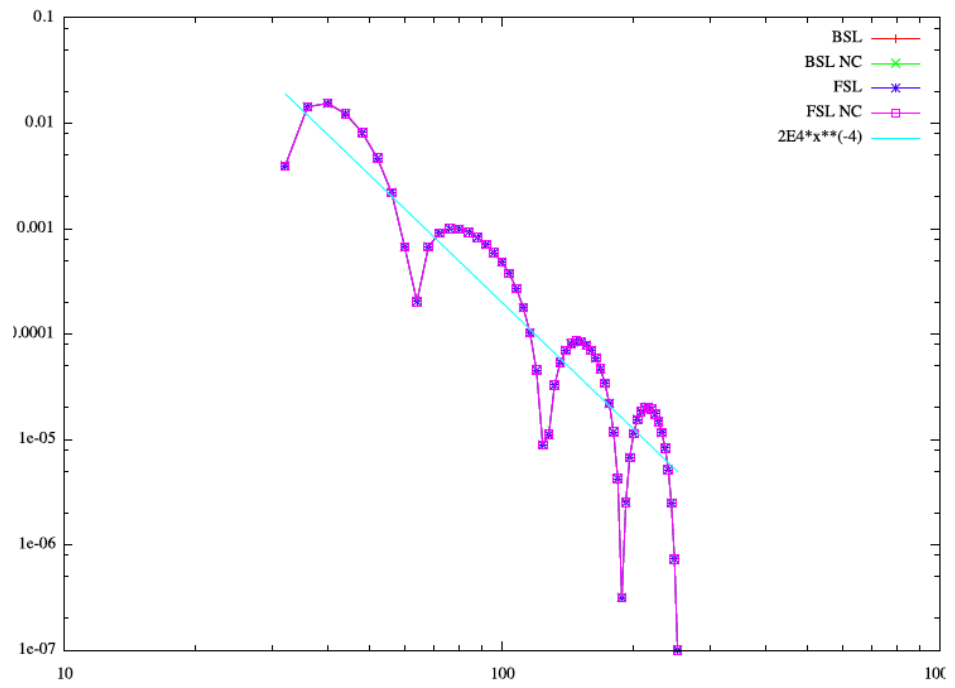




\section{Case of rotation polar coordinates $\Delta t=0.1$}

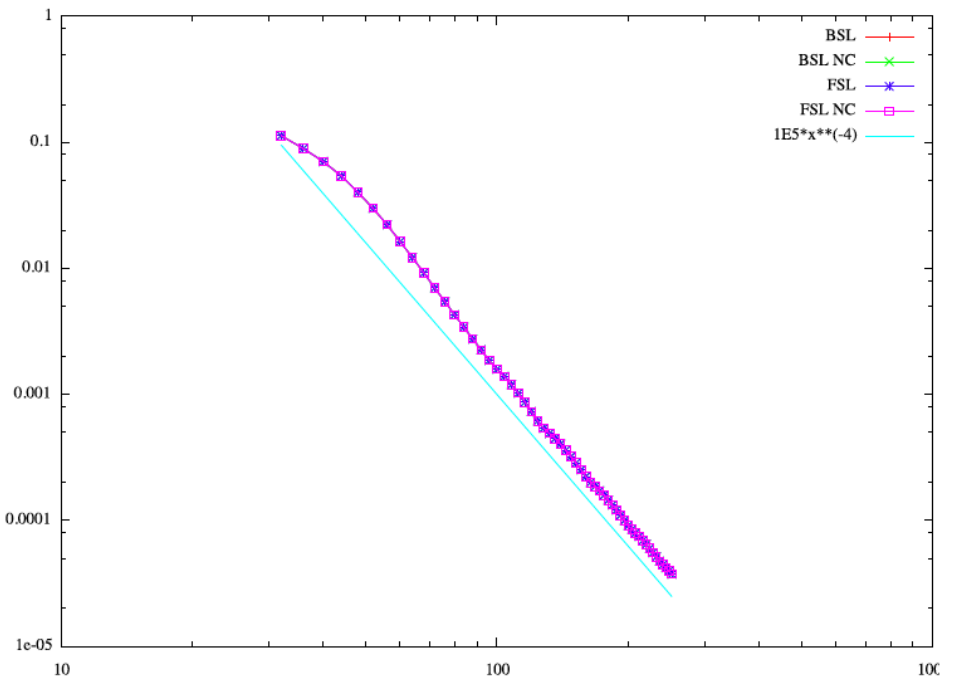




\section{Case of translation polar coordinates $\Delta t=0.1 \Delta x$}

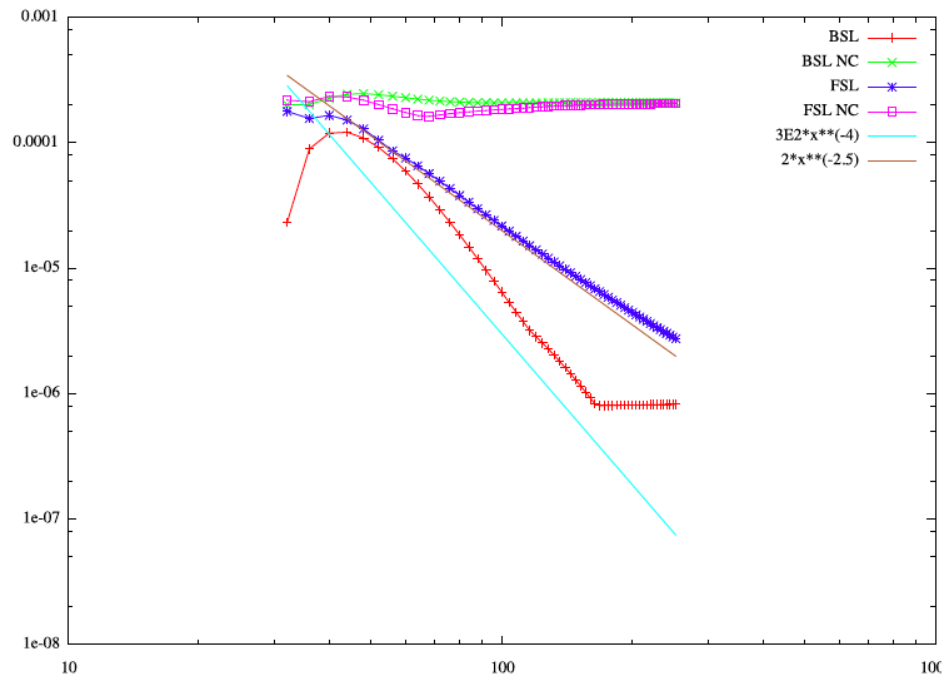




\section{Case of translation polar coordinates $\Delta t=0.1$}

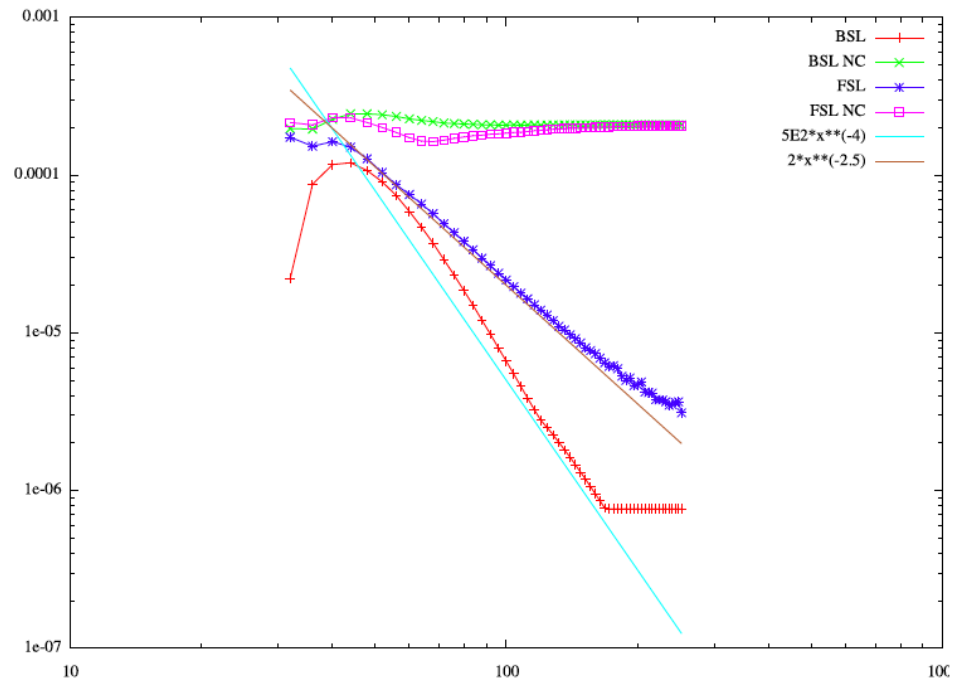




\section{Case of complex field polar coordinates $\Delta t=0.1 \Delta x$}

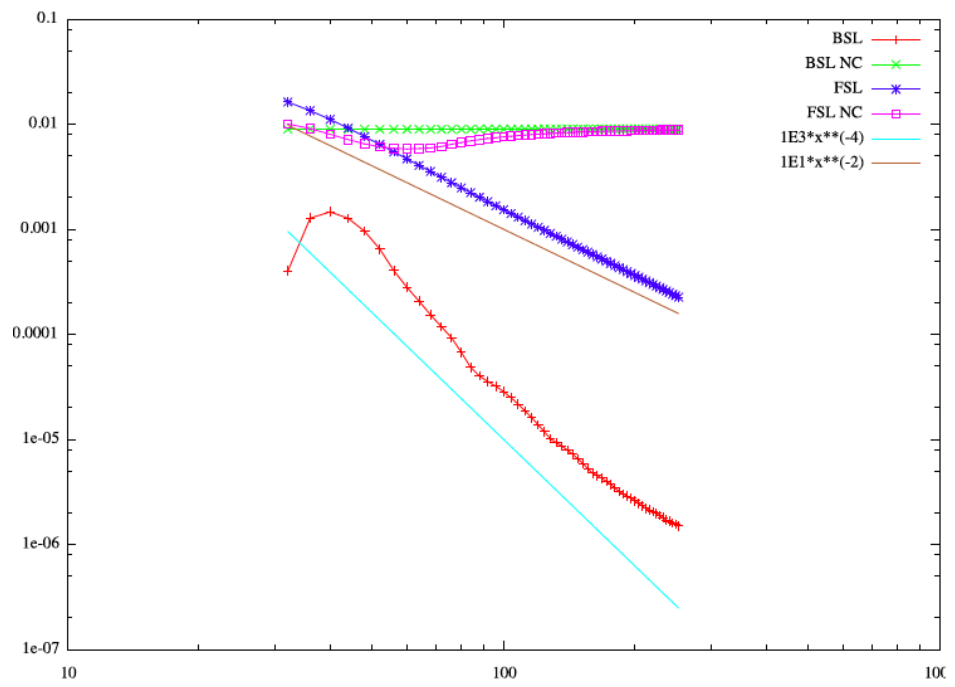




\section{Case of complex field polar coordinates $\Delta t=0.1$}

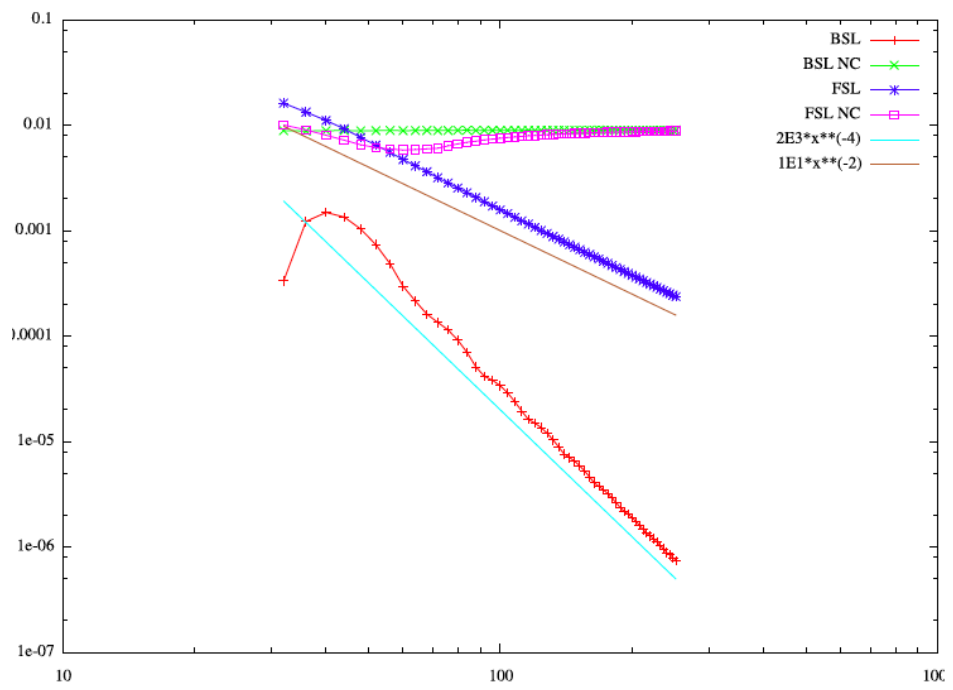




\section{Cartesian case anisotropic rotation $\Phi=4(x-x 0)^{2}+(y-y 0)^{2} d y=d x$}

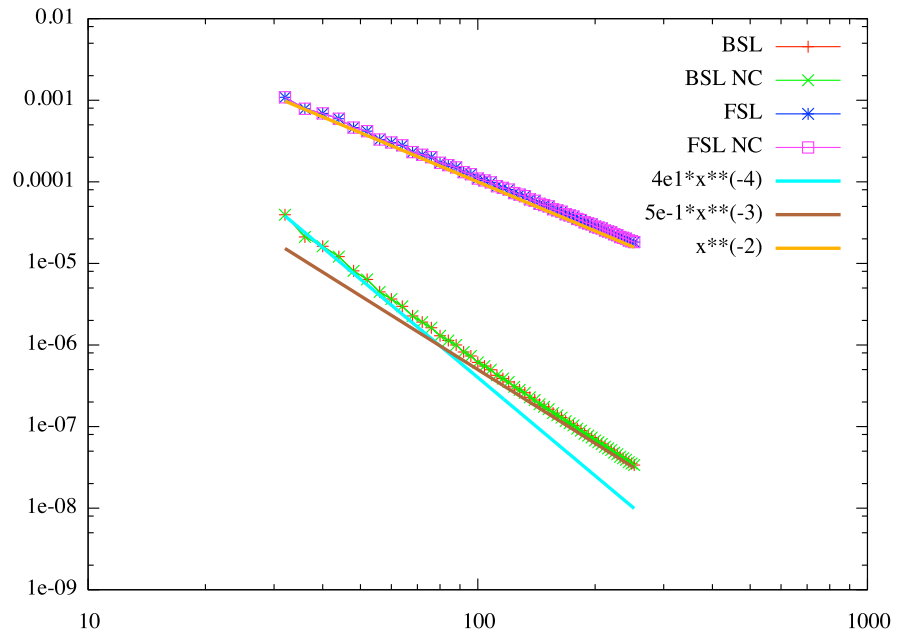




\section{Cartesian case anisotropic rotation

$$
\Phi=4(x-x 0)^{2}+(y-y 0)^{2} d y=2 d x
$$

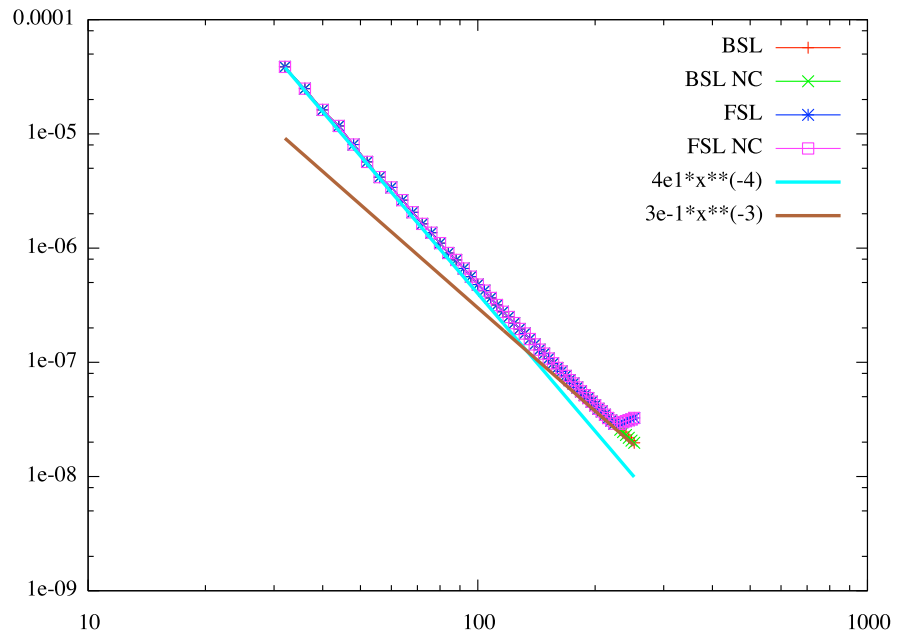




\section{GYSELA 4D toric case $\mu=0$ mass BSL/FSL}

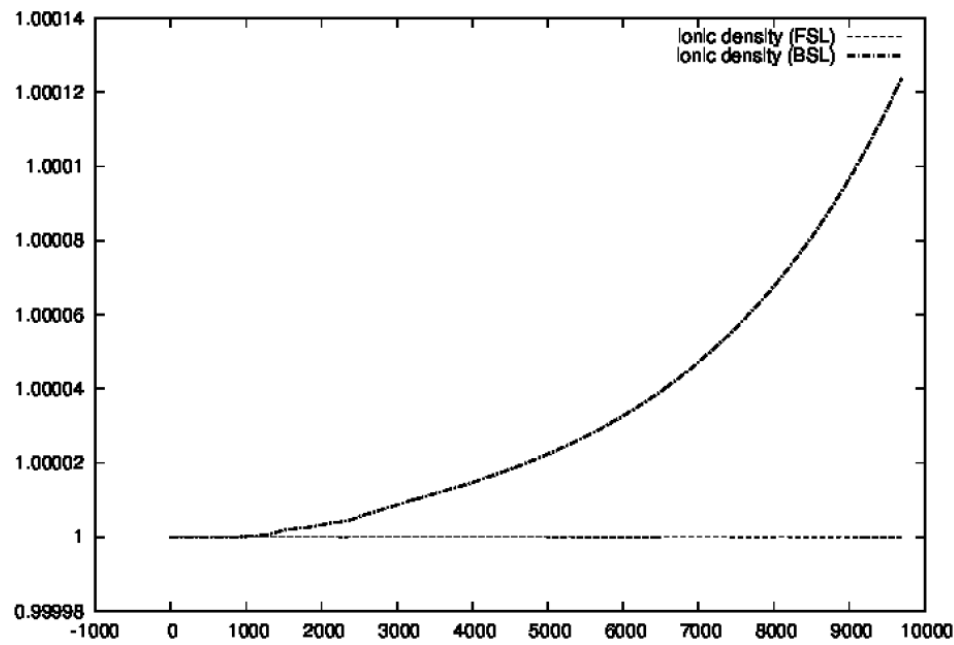




\section{GYSELA 4D toric case $\mu=0$ energy FSL}

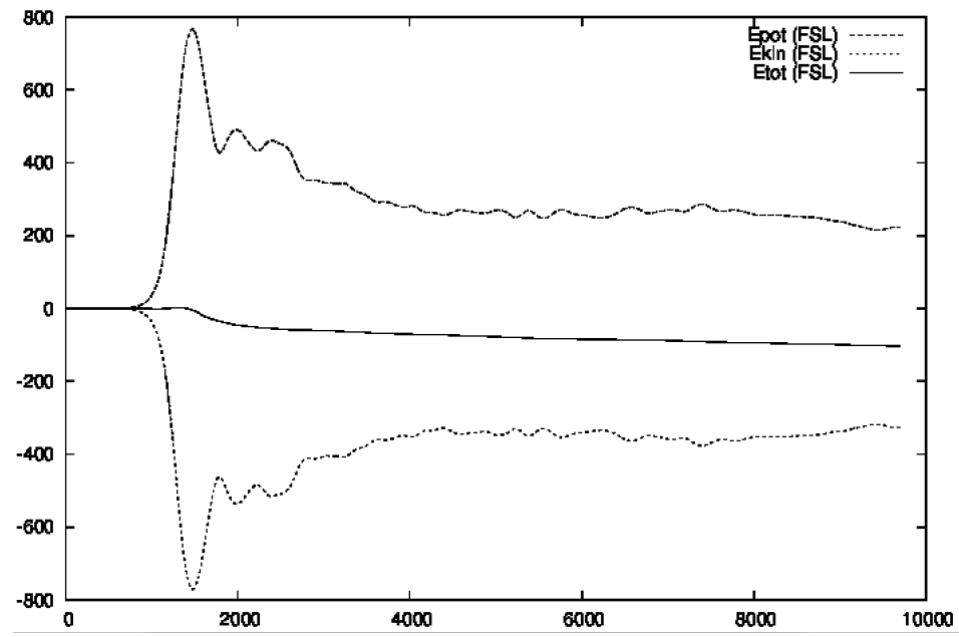




\section{GYSELA 4D toric case $\mu=0$ energy BSL}

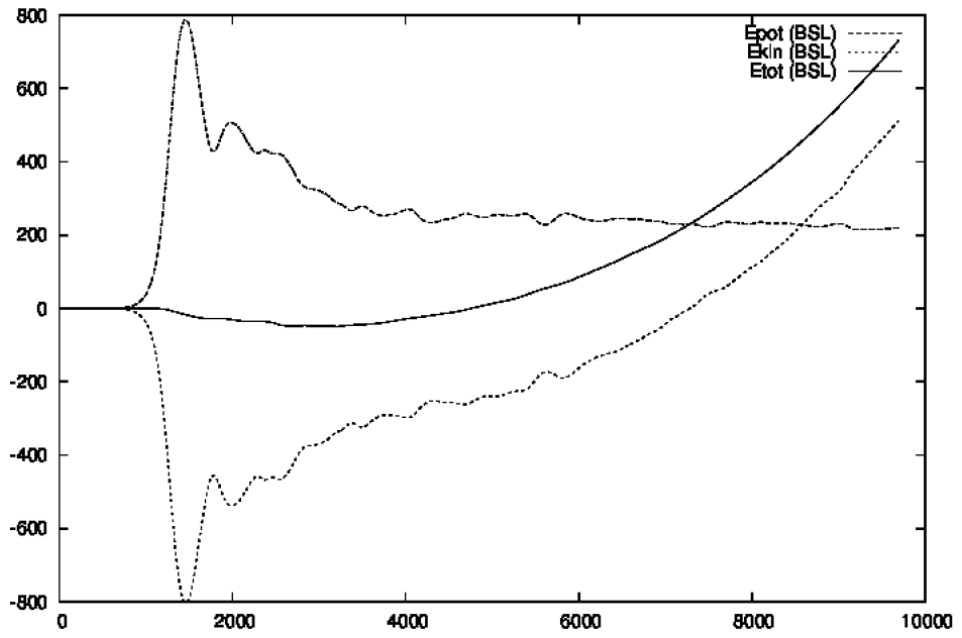




\section{The equation}

$$
\partial_{t} \bar{f}+\partial_{\eta^{1}}\left(\frac{\partial_{\eta^{2}} \Psi}{\sqrt{g}} \bar{f}\right)+\partial_{\eta^{2}}\left(-\frac{\partial_{\eta^{1}} \Psi_{\bar{f}}}{\sqrt{g}}\right)=0,
$$

with $\bar{f}=\sqrt{g} \tilde{f}, \tilde{f}\left(\eta^{1}, \eta^{2}\right)=f\left(x_{1}\left(\eta^{1}, \eta^{2}\right), x_{2}\left(\eta^{1}, \eta^{2}\right)\right)$ and

$$
\sqrt{g}=\partial_{\eta^{1}} x_{1} \partial_{\eta^{2}} x_{2}-\partial_{\eta^{2}} x_{1} \partial_{\eta^{1}} x_{2} \text {. }
$$




\section{The CSL method}

1. Split by direction

2. Start from

$$
\frac{1}{\Delta \eta^{1}} \int_{\eta_{i}^{1}}^{\eta_{i+1}^{1}} \bar{f}_{j}^{n}\left(\eta^{1}\right) d \eta^{1}=\frac{1}{\Delta \eta^{1}} \int_{\xi_{i}^{1}}^{\xi_{i+1}^{1}} \tilde{f}_{j}^{n}\left(\eta^{1}\left(\xi^{1}\right)\right) d \xi^{1}
$$

3. Update through

$$
\frac{1}{\Delta \eta^{1}} \int_{\xi_{i}^{1}}^{\xi_{i+1}^{1}} \tilde{f}_{j}^{n+1}\left(\eta^{1}\right) d \eta^{1}=\frac{1}{\Delta \eta^{1}} \int_{\xi_{i, j}^{1 *}}^{\xi_{i+1, j}^{*}} \tilde{f}_{j}^{n}\left(\eta^{1}\left(\xi^{1}\right)\right) d \xi^{1}
$$

4. The $\left(\xi_{i}^{1}\right)$ mesh is non uniform; use of cubic splines on non uniform mesh 


\section{Fondamental property}

For

$$
\tilde{\Psi}\left(t, \eta_{1}, \eta_{2}\right)=H=\eta_{1},
$$

we get

$$
\xi_{i, j}^{1 *}=\xi_{i}^{1}, \xi_{j, i}^{2 *}=\xi_{j}^{2}+\Delta t
$$

A function constant in $\eta_{1}$ remains constant in the numerical scheme 


\section{Example of mesh}

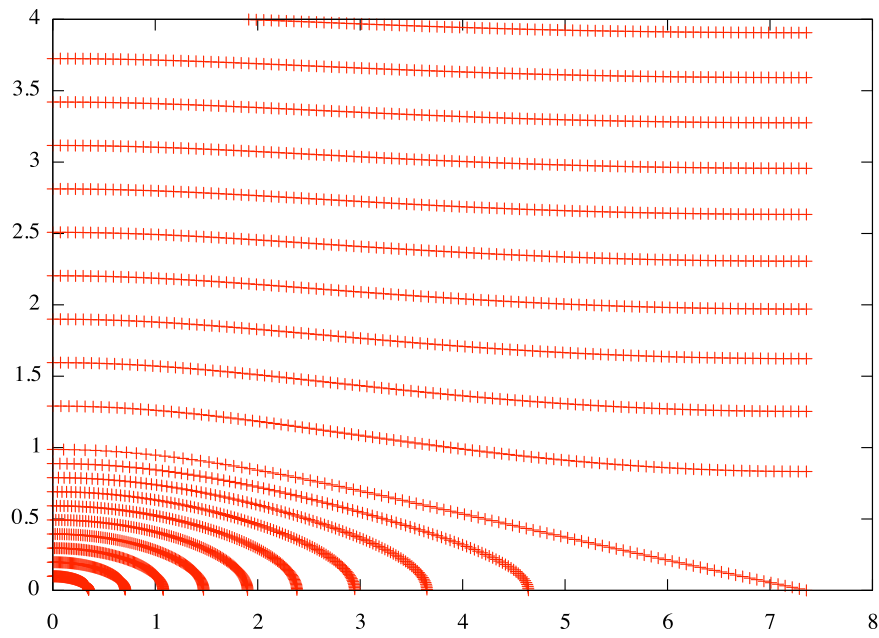




\section{Example of mesh}

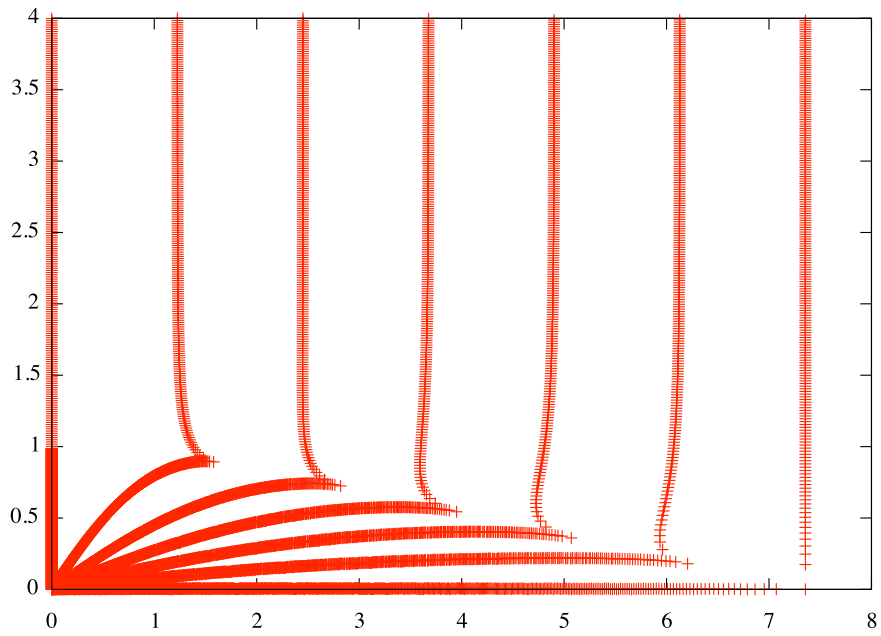




\section{Example of mesh}

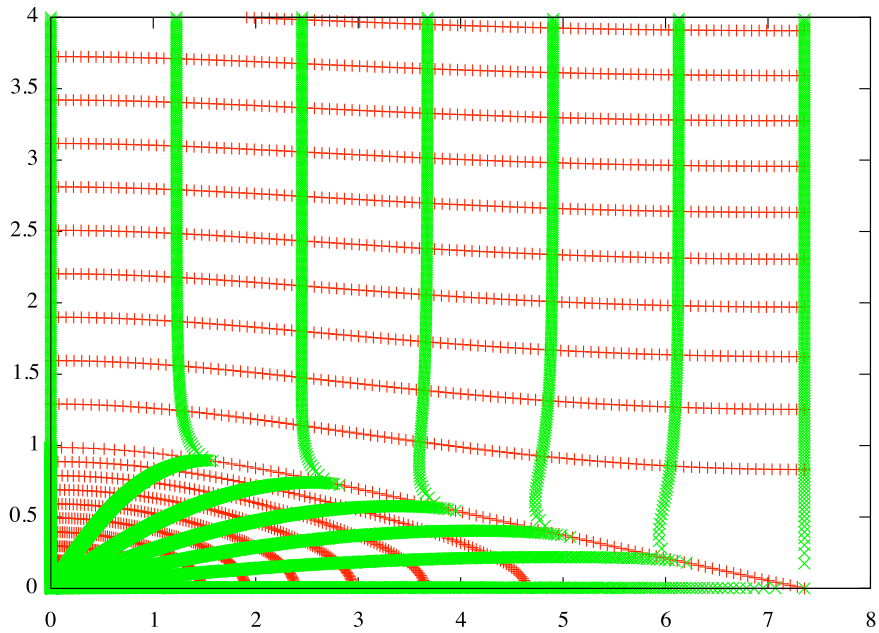




\section{First tests on Colella mesh $\alpha=1 e-2$}

DB: mesh.xmf Time:0 Mesh
Vor: mesh
ond

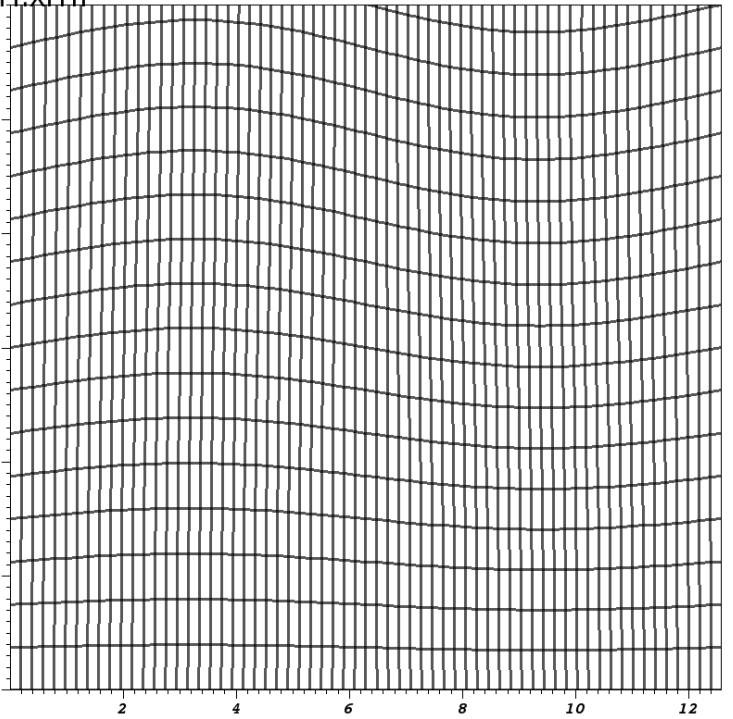




\section{First tests on Colella mesh $\alpha=1 e-2$}

DB: mesh.xmf Time:0

Mesh
Vor: mesh
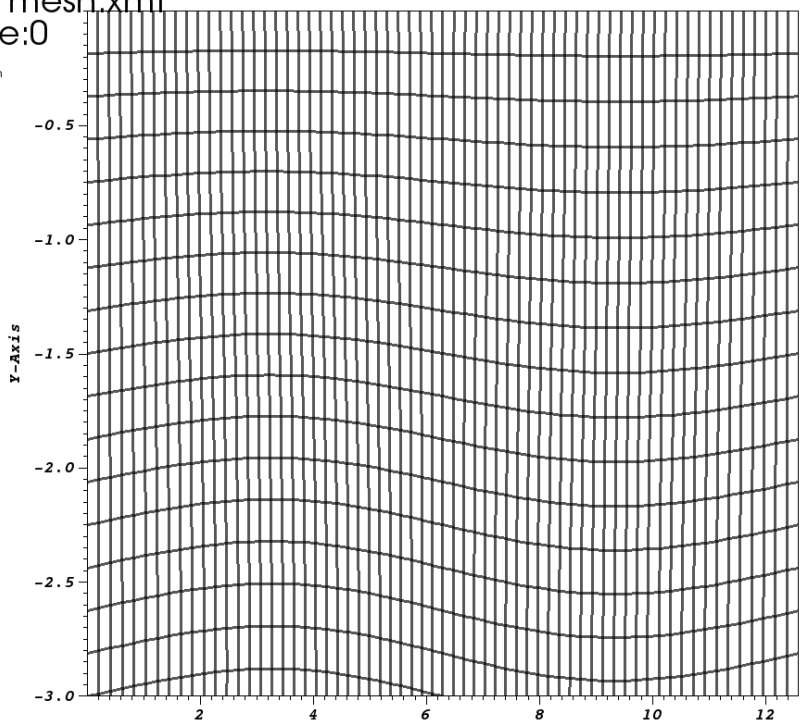


\section{First tests on Colella mesh $\alpha=1 e-1$}

DB: mesh.xmf Time:0

Mesh
Var: mesh
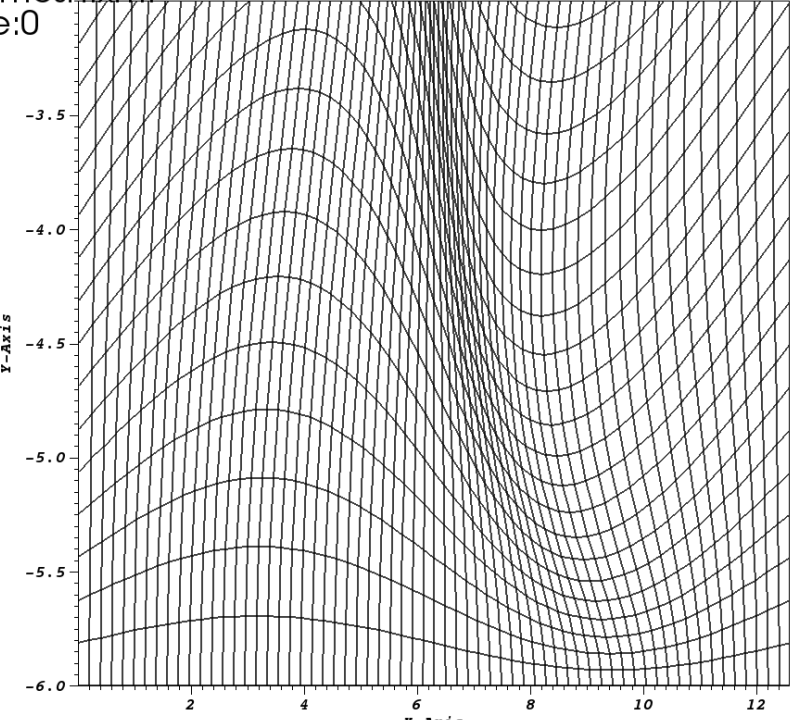


\section{First tests on Colella mesh $\alpha=1 e-1$}

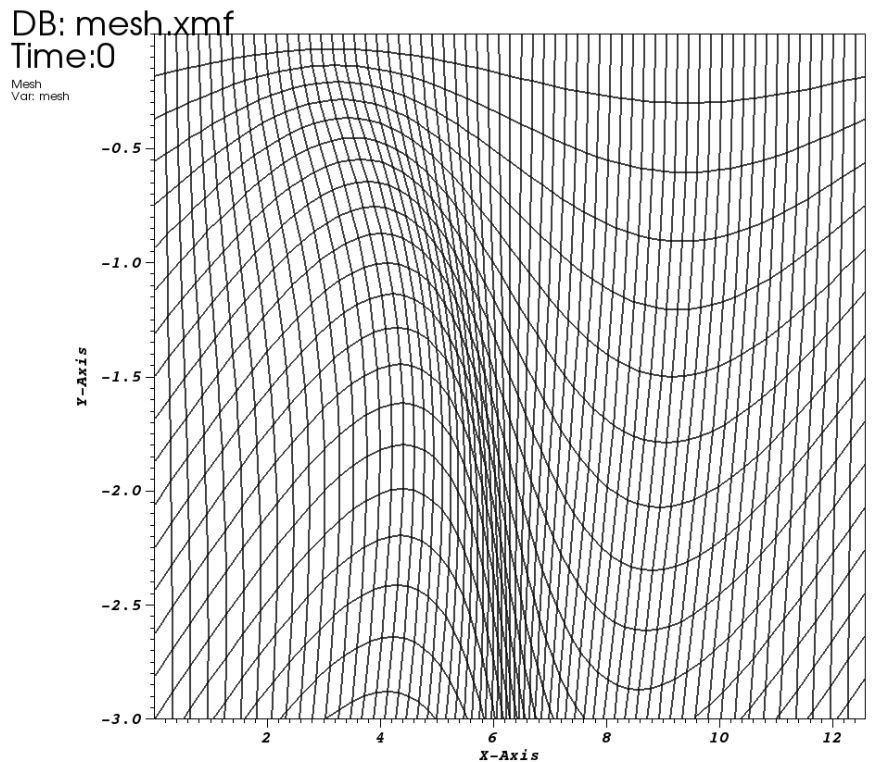




\section{Conservative Finite Difference method}

Comparison with a conservative finite difference method (CFD)

1. High order reconstruction

2. CFL restriction

3. divergence free preservation, for centered reconstruction 


\section{Linear Landau damping $N=64$}

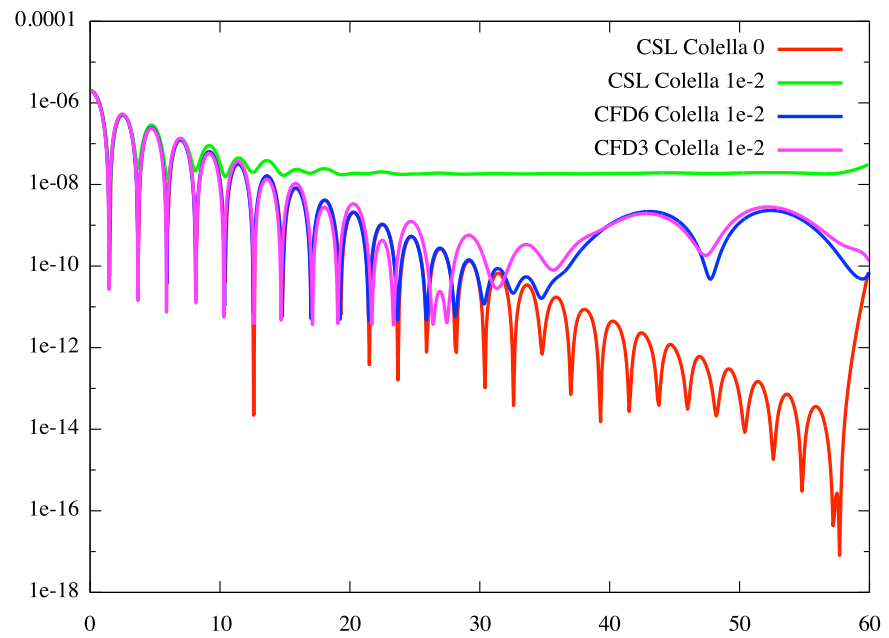




\section{Linear Landau damping $N=64$}

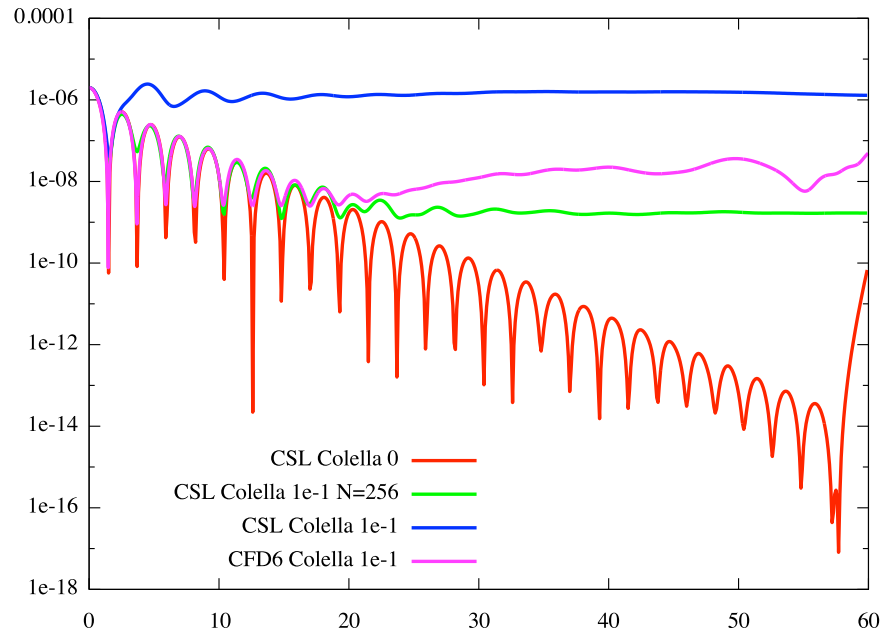




\section{Conclusion/Perspectives}

1. Highlighting of fondamental properties : mass, divergence free, high order

2. Difficulties to have everything in the Semi-Lagrangian framework

3. Mixing strategies : decouple known linear displacement and small non linear displacement 